EDITORIAL

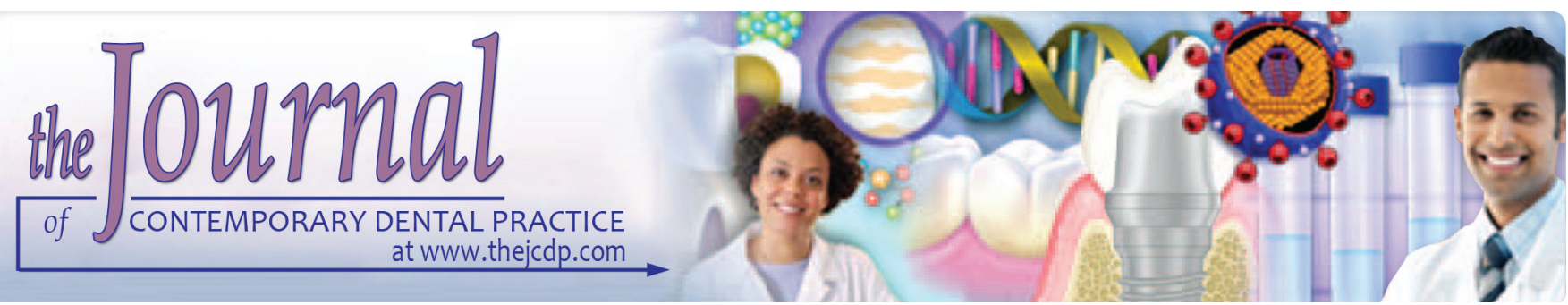

\title{
New Directions for Oral Submucous Fibrosis Research: Whole Evaluation for Holistic Rehabilitation!
}

\author{
${ }^{1}$ Prashanth Panta, ${ }^{2}$ Sachin C Sarode, ${ }^{3}$ Gargi S Sarode, ${ }^{4}$ Shankargouda Patil
}

\begin{abstract}
Oral submucous fibrosis (OSF) is an oral potentially malignant disorder (OPMD) associated with prolonged betel-quid (BQ) use. It affects both physiological and psychological dimensions, leading to a significant morbidity. Some challenges in OSF research include: Assessment and correction of: (i) Quality of life, (ii) burning sensation, and (iii) nutritional deficiency. There are numerous medical treatments for OSF, but most of them fail to offer complete rehabilitation. This is because some underpinnings of this condition like "mental factors" that contribute to habit continuation at root level, "poor quality of life," and "nutritional deficiencies" are still uncorrected. We believe that OSF must be managed at the "mind and body" level to accomplish holistic rehabilitation.

Herein, we briefly describe the general condition of OSF patients, and highlight the possibility of a novel telomere-based assessment of psychosocial stress, and support use of meditation to counter excessive "telomere shortening" which is linked to oral cancer. We also expand the current view on "burning sensation" in OSF from an entire physical concept to a possibility of mental factors, and finally recommend rich, micronutrient supplementation (through diet) to counter free radical production within the hypoxic oral epithelium.
\end{abstract}

Keywords: Betel-quid, Meditation, Mind, Quality-of-life.

How to cite this article: Panta P, Sarode SC, Sarode GS, Patil S. New Directions for Oral Submucous Fibrosis Research:

\footnotetext{
${ }^{1}$ Department of Oral Medicine and Radiology, MNR Dental College \& Hospital, Sangareddy, Telangana, India

${ }^{2,3}$ Department of Oral Pathology and Microbiology, Dr. D. Y. Patil Dental College \& Hospital, Dr. D.Y. Patil Vidyapeeth, Pune Maharashtra, India

${ }^{4}$ Division of Oral Pathology, Department of Maxillofacial Surgery and Diagnostic Sciences, College of Dentistry, Jazan University Jazan, Kingdom of Saudi Arabia

Corresponding Author: Prashanth Panta, Department of Oral Medicine and Radiology, MNR Dental College \& Hospital Sangareddy, Telangana, India, Phone: +919701806830, e-mail: maithreya.prashanth@gmail.com
}

Whole Evaluation for Holistic Rehabilitation! J Contemp Dent Pract 2018;19(8):901-903.

Source of support: Nil

Conflict of interest: None

\section{PHYSIOLOGICAL IMPAIRMENT OUTSIDE ORAL CAVITY}

Oral submucous fibrosis is no more a localized condition, but a regional and systemic one. Fibrosis in OSF can often extend from the oral cavity to the oropharynx, and up to the esophagus in some cases, and the toxic effects involve multiple organ systems. Betel-quid users have shown systemic complications ranging from tachycardia, arterial stiffness, poor working memory and motor performance, and substantial functional connectivity changes like white-matter deficit in brain. ${ }^{1-3}$ Betel nut is a potential mixture of numerous harmful alkaloids bearing a serious threat to the general health of all patients.

\section{ASSESSMENT AND CORRECTION OF PSYCHOSOCIAL IMPAIRMENT}

Psychosocial impairment is very common in OSF patients. The symptoms "burning sensation" and "difficulty in mouth opening" (approaching $<1 \mathrm{~cm}$ ) are the main reason for chronic stress and depression in these patients. Furthermore, these patients may participate in emotional chewing of $\mathrm{BQ}$ due to work-stress and life-stress, and additionally, patients develop "dependence" due to the addictive nature of $\mathrm{BQ}$ ingredients. Overall, both patientrelated factors (primarily mental) and substance-related factors form a vicious cycle and result in a debilitated final state characterized by $\mathrm{BQ}$ overuse, increasing the severity of OSF, and risk of malignancy. Therefore, to counter a complex habit like BQ use, we recommend mind management alongside habit discontinuation and medical management of existing OSF symptoms. 


\section{Telomere: A Powerful Marker to assess Psychosocial State}

Lifestyle factors are deeply associated with telomerase activity and telomere length. Authors have often recorded changes in cortisol levels in OSF, considering cortisol as a marker of psychological stress. "Telomerase length" is another reliable marker of psychosocial condition in OSF. A short telomere is a metric for debilitated/weakened mental condition, and is often associated with high scores of "stress and depression", which are noticed in many OSF patients. ${ }^{4}$ Therefore, telomere length can be a true test of psychological morbidity in OSF. So far, not a single report exists on the assessment of telomeres in the context of assessing psychosocial stress. Bau et al. showed shortest telomere length in oral squamous cell carcinoma, intermediate length in OPMDs, and longest in healthy controls, indicating declining telomere length to have direct implications in oral cancer. ${ }^{5}$ Many authors often combine OPMDs into one group during assessment of psychological stress, which itself is a confounding factor. The OSF patients may be subject to higher psychosocial stress compared to other OPMDs like leukoplakia, oral lichen planus, and erythroplakia because, it is the symptoms burning sensation and difficulty in mouth opening in OSF that hamper quality-of-life.

\section{Meditation Intervention Lengthens Telomeres}

Meditation can impact BQ overuse in the context of "habit breaking" and by "induction of self-control." Another important effect of meditation is its potential to lengthen telomeres, ${ }^{6}$ adding a newer perspective for overall improvement in OSF though meditation. Shortened telomeres are linked to several chronic diseases and many cancers including oral cancer. ${ }^{5}$ We presume that meditation can benefit BQ users and OSF patients additionally through the "telomere pathway," reducing risk of oral cancer. Telomere length is influenced by the neuro-endocrine hormone "cortisol" also elevated in OSF. ${ }^{7}$ Meditation reduces cortisol levels by balancing sympathetic-parasympathetic response and induces selfcontrol through its workings on the brain, reducing the overall dependence on $B Q$, and arresting development of severe form of OSF.

We presume the determination of "telomere length" in OSF can help us capture the subtle levels of psychosocial stress at the root level of this condition. Moreover, the possible role of mind-body interventions which we proposed earlier for BQ users and OSF patients ${ }^{8,9}$ may further lengthen telomeres and improve health at cellular scale. Another mode of action of mindfulness practices is their effect on the nuclear factor kappa beta (NF-K $\beta$ ) pathway. In OSF, there is strong inflammatory infiltrate which may be also linked to the symptom "burning sensation" at gross level, and cancer development at the fundamental level. A downregulation of inflammatory pathway NF-K $\beta$ may prevent oral cancer development within OSF! The cellular pivots like telomere length and NF-K $\beta$ pathway are among the determinants of cancer development. The "cellular-rehabilitation effect" induced by meditation practice is essential for OPMDs like OSF which bear substantial risk of progression to malignancy.

\section{Burning Sensation may have Its Roots in the Mind}

Concomitant burning mouth syndrome was reported previously in a small fraction of OSF cases. ${ }^{10}$ Burning sensation in OSF is due to erosive and atrophic changes following mucosal trauma from BQ chewing and due to betel nut alkaloids. Besides this, decrease in secretion of salivary mucins from minor salivary glands was also hypothesized to cause burning sensation. ${ }^{11}$ Burning sensation can also arise due to the deficiency in B12 and other essential micronutrients. We believe "burning sensation" in OSF may be also be linked to the degree of "psychological morbidity". Based on recent reports which explored neurological and psychological morbidity in OSF patients, we presume a strong contribution of mental factors to the cumulative scores of burning sensation. Similar to burning mouth syndrome, the central neuropathic mechanisms may be parallel players in OSF.

Generally speaking, burning sensation is a complex event driven many times by "local factors," and in the absolute absence of local factors "neurogenic factors" are to be blamed. Burning sensation during early stages of OSF brings us close to possible central mechanisms within the nervous system. Also, OSF showed relief with alphalipoic acid (ALA), a medical treatment for burning mouth syndrome. In a small study by Rao, ${ }^{12}$ addition of ALA to intralesional steroid and hyaluronidase improved burning mouth sensation and mouth opening. It is important to understand how much burning sensation in OSF patients is precipitated by local factors like epithelial atrophy, and how much is due to the involvement of neurogenic factors. We believe there is a confusing overlap of both local and mental factors in the genesis of burning sensation in OSF.

\section{Micronutrient Supplementation may counter High-risk Reactive Oxygen Species}

Oral submucous fibrosis is a condition demonstrating serious free radical production within the oral epithelium, due to the existence of a fibrosis barrier between the vasculature and epithelium. "Continuous hypoxia across the oral epithelium" is well known to generate a wide range of free radicals which increase risk of malignancy. As a 
result, free radical quenchers like vitamins $\mathrm{C}$ and $\mathrm{E}$, and other powerful antioxidants like $\beta$-carotenes, lycopene, and spirulina supplementation can be helpful.

It is essential to correct levels of depleting micronutrients (both vitamins and minerals) because the micronutrients deficient in OSF normally exert a protective effect against cancers. Moreover, adding vitamins (A, B complex, C, D, and E) and minerals (iron, calcium, copper, zinc, magnesium, etc.) to the treatment regimen of OSF improved clinical response (intolerance to spicy food, burning sensation, and also mouth opening). ${ }^{13}$ Deficiency in vitamin B12 and folate levels could be due to gastric parietal cell antibodies seen in OSF patients. ${ }^{14}$ Some of the systemic manifestations like poor memory, stress and depression, and weakness may be consequences of deficiency in these essential micronutrients (vitamin B12, folate, iron deficiency, etc.).

As OSF is a chronic condition, we believe it is wise to rely on foods rich in free radical quenching antioxidants more than external supplementation. A diet rich in essential micronutrients can benefit OSF patients preventing malignant transformation. To benefit patients with OSF, ideally, we must fuse "habit discontinuation" and "medications" (conventional thought) with "mind-body practices" like meditation to achieve full rehabilitation, as OSF has roots in the mind, and ramifications in the body.

\section{REFERENCES}

1. Wei YT, Chou YT, Yang YC, Chou CY, Lu FH, Chang CJ, Wu JS. Betel nut chewing associated with increased risk of arterial stiffness. Drug Alcohol Depend 2017 Nov;180:1-6.

2. Yuan F, Zhu X, Kong L, Shen H, Liao W, Jiang C. White matter integrity deficit associated with betel quid dependence. Front Psychiatry 2017 Oct;8:201.

3. Osborne PG, Chou TS, Shen TW. Characterization of the psychological, physiological and EEG profile of acute betel quid intoxication in naïve subjects. PLoS One 2011 Aug;6(8):e23874.
4. Kanodia S, Giri VP, Giri OP, Devi MP, Garima Y. Assessment of anxiety, depression, and serum cortisol level in oral submucous fibrosis patients: a controlled clinical trial. Eur J Dent 2017 Jul-Sep;11(3):293-298.

5. Bau DT, Lippman SM, Xu E, Gong Y, Lee JJ, Wu X, Gu J. Short telomere lengths in peripheral blood leukocytes are associated with an increased risk of oral premalignant lesion and oral squamous cell carcinoma. Cancer 2013 Dec;119(24):4277-4283.

6. Jacobs TL, Epel ES, Lin J, Blackburn EH, Wolkowitz OM, Bridwell DA, Zanesco AP, Aichele SR, Sahdra BK, MacLean KA, et al. Intensive meditation training, immune cell telomerase activity, and psychological mediators. Psychoneuroendocrinology 2011 Jun;36(5):664-681.

7. Steptoe A, Hamer M, Lin J, Blackburn EH, Erusalimsky JD. The longitudinal relationship between cortisol responses to mental stress and leukocyte telomere attrition. J Clin Endocrinol Metab 2017 Mar;102(3):962-969.

8. Sarode SC, Panta P, Sarode GS, Gadbail AR, Gondivkar SM, Patil S. New research directions for areca nut/betel quid and oral submucous fibrosis for holistic prevention and treatment. Oral Oncol 2018 Mar;78:218-219.

9. Panta P, Sarode SC, Sarode GS, Gadbail AR, Gondivkar SM, Patin S. "Mind" in betel-quid use and related disorders. J Contemp Dent Pract 2018 Jun;19(6):1-2.

10. Sarode SC, Sarode GS. Primary burning mouth syndrome in oral submucous fibrosis patients. Oral Oncol 2011 Jul;47(7):683.

11. Sarode SC, Sarode GS. Burning sensation in oral submucous fibrosis and its possible association with mucin secreted by affected minor salivary glands. Oral Oncol 2013 Apr;49(4):e16-e17.

12. Rao PK. Efficacy of alpha lipoic acid in adjunct with intralesional steroids and hyaluronidase in the management of oral submucous fibrosis. J Cancer Res Ther 2010 Oct-Dec;6(4):508-510.

13. Maher R, Aga P, Johnson NW, Sankaranarayanan R, Warnakulasuriya S. Evaluation of multiple micronutrient supplementation in the management of oral submucous fibrosis in Karachi, Pakistan. Nutr Cancer 1997;27(1):41-47.

14. Wang YP, Wu YC, Cheng SJ, Chen HM, Sun A, Chang JY. High frequencies of vitamin B12 and folic acid deficiencies and gastric parietal cell antibody positivity in oral submucous fibrosis patients. J Formos Med Assoc 2015 Sep;114(9):813-819. 